\title{
Efficiency Scaling of High Harmonic Generation driven by a tunable Optical Parametric Amplifier in the Visible
}

\author{
Giovanni Cirmi ${ }^{1,2,}$, Chien-Jen Lai ${ }^{1}$, Eduardo Granados ${ }^{1,3}$, \\ Shu-Wei Huang ${ }^{1}$, Phillip Keathley ${ }^{1}$, Alexander Sell ${ }^{1}$, and F. X. Kärtner ${ }^{1,2}$, \\ ${ }^{1}$ Department of Electrical Engineering and Computer Science and Research Laboratory of Electronics, \\ Massachusetts Institute of Technology,77 Massachusetts Ave,Cambridge, MA 02139, USA \\ ${ }^{2}$ Center for Free-Electron Laser Science, DESY and University of Hamburg, Notkestraße 85, D-22607 Hamburg, Germany \\ ${ }^{3}$ IKERBASQUE, Basque Foundation for Science, 48011, Bilbao, Spain \\ *giocirmi@mit.edu
}

\begin{abstract}
High Harmonic Generation efficiency increases for short driver wavelengths. We study experimentally the driver wavelength dependence around $32 \mathrm{eV}$ by driving the process with a tunable Optical Parametric Amplifier in the visible range.

OCIS codes: (190.0190) Nonlinear Optics; (190.4970) Parametric oscillators and amplifiers; (190.2620) Harmonic generation and mixing.
\end{abstract}

\section{Introduction}

High Harmonic Generation (HHG) is an optical technique allowing for the production of photons in the Extreme UV (EUV), soft and hard X-ray regions, obtained by focusing an ultraviolet, visible or infrared femtosecond pulse, called driver, in a material, typically a noble gas. HHG efficiency and cutoff extension have a strong dependence on the wavelength of the driver pulse. In this work we demonstrate a high energy tunable Optical Parametric Amplifier (OPA) in the visible range. With this source we generate HHG in Argon, study the wavelength dependency of the energy conversion efficiency for single harmonics, and find good agreement with a previously developed theory.

\section{Tunable OPA in the visible}

The OPA used is based on the work in reference [1]. The setup is shown in Fig. 1. We start from a commercial amplified Ti:sapphire laser emitting $6 \mathrm{~mJ}, 35$-fs pulses at $800 \mathrm{~nm}$ with a 1-kHz repetition rate. The amplifier output energy is split into three parts. $1 \%$ of the pulse energy is used to generate a seed in the visible range by white light continuum generation in a 2-mm-long sapphire plate. $9 \%$ of the energy is frequency doubled in a 0.5 -mm long $\beta$ Barium Borate (BBO) crystal, generating a $40 \mu \mathrm{J}$ pump at $400 \mathrm{~nm}$ used to amplify the visible seed up to $4 \mu \mathrm{J}$ in a 1 $\mathrm{mm}$-long BBO crystal. A SF10 prism compressor, with 7-cm apex distance, is used to compensate for the dispersion induced by the materials crossed. The remaining $90 \%$ of the amplifier output energy is frequency doubled and generates $2 \mathrm{~mJ}$ pulses at $400 \mathrm{~nm}$. These pulses are used as a pump for the second and third OPA stages. The first OPA stage energy is amplified to around $15-20 \mu \mathrm{J}$ in a 1-mm-long BBO crystal (second OPA stage), and the output is amplified in the third stage up to $200 \mu \mathrm{J}$. To improve the wavefront matching, we tilt the pump wavefront with a $\mathrm{CaF}_{2}$ prism.

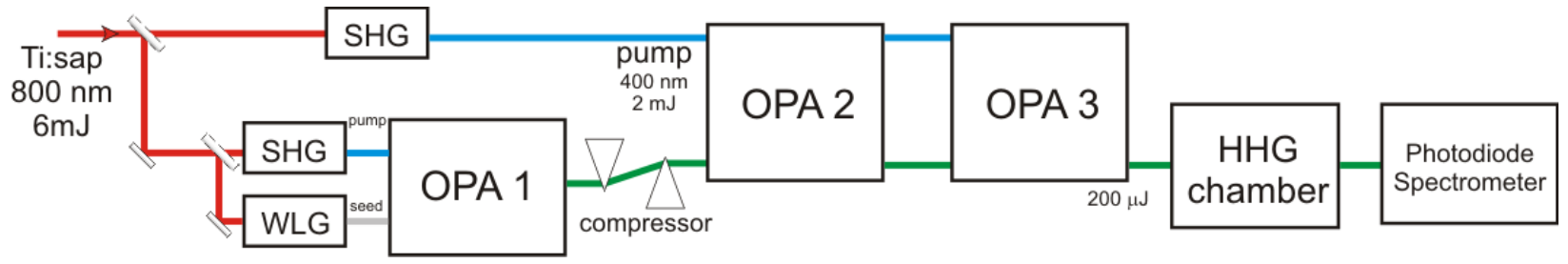

Fig. 1: schematic of the experimental setup.

The OPA shows a broad tunability in the visible range, from $500 \mathrm{~nm}$ to $630 \mathrm{~nm}$. We characterized the OPA output duration by self diffraction background free autocorrelation, and the Full Width at Half Maximum (FWHM) duration is between 34 and 39 fs. Pulses shorter than 6 fs could be obtained by using a double chirped mirror compressor instead of a prism compressor [2]. The pulse-to-pulse energy stability is $2.54 \% \mathrm{rms}$, measured over 9 minutes. The OPA shows very good noise performances, with a superfluorescence level below $0.5 \%$ of the signal energy. The beam $\mathrm{M}^{2}$ is 1.9 . 

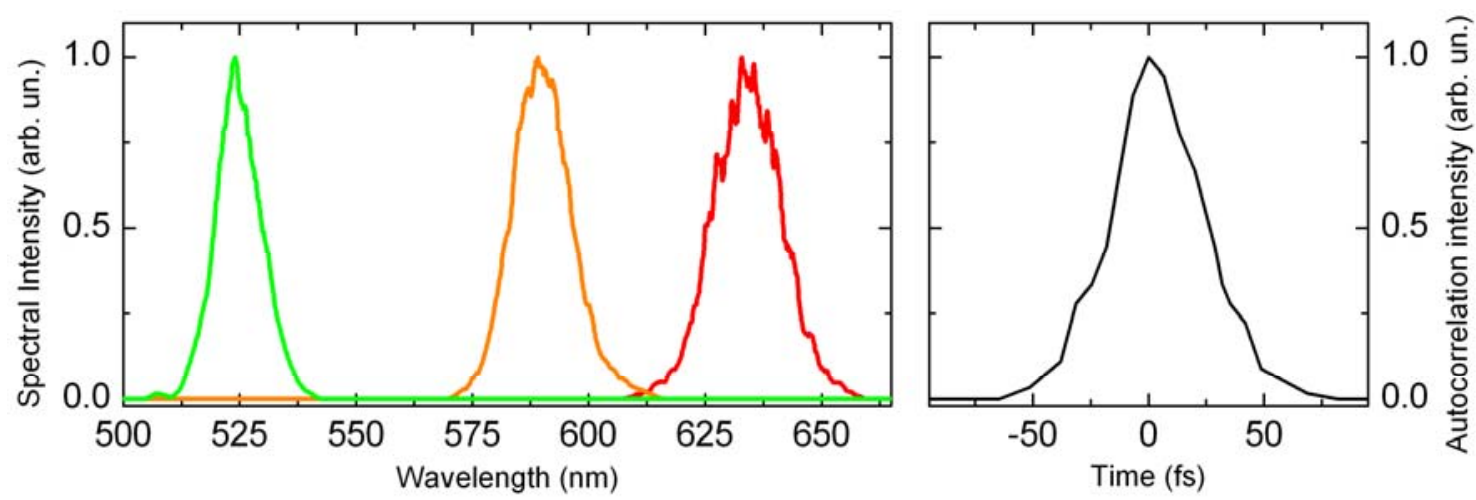

Fig. 2: Spectra and autocorrelation of the visible OPA. The spectra shown were used to drive the HHG experiment. The autocorrelation is relative to the $590 \mathrm{~nm}$ pulse and corresponds to $34 \mathrm{fs}$ FWHM pulse duration.

\section{HHG in Argon}

We used the OPA to drive HHG in an Argon gas jet, with a 40 mbar pressure (500 mbar backing pressure), and measure the efficiency around $32 \mathrm{eV}$. We drive HHG with 3 wavelengths generated by the OPA, namely $524 \mathrm{~nm}$, $589 \mathrm{~nm}$ and $633 \mathrm{~nm}$, and with the fundamental and second harmonic frequencies of the laser source, i.e. $800 \mathrm{~nm}$ and $400 \mathrm{~nm}$. The spectra of the OPA and one of the autocorrelations are shown in Fig. 2. We measure the beam size in the focal position with the knife edge method and keep similar intensities for all 5 driver wavelengths. Table 1 summarizes the characteristics of the 5 driver pulses.

Table 1: Characteristics of the driver pulses used for the HHG wavelength scaling study.

\begin{tabular}{|l|l|l|l|l|}
\hline $\begin{array}{l}\text { Driver wavelength } \\
(\mathrm{nm})\end{array}$ & $\begin{array}{l}\text { Duration FWHM } \\
(\mathrm{fs})\end{array}$ & $\begin{array}{l}\text { Size at focus } \\
(\mu \mathrm{m} \times \mu \mathrm{m})\end{array}$ & Energy $(\mu \mathrm{J})$ & $\begin{array}{l}\text { Intensity } \\
\left(\mathrm{x} 10^{14} \mathrm{~W} / \mathrm{cm}^{2}\right)\end{array}$ \\
\hline 400 & 26 & $26 \times 26$ & 85 & 3.1 \\
\hline 524 & 39 & $33 \times 23$ & 82 & 1.8 \\
\hline 589 & 34 & $34 \times 17$ & 85 & 2.8 \\
\hline 633 & 36 & $31 \times 18$ & 82 & 2.6 \\
\hline 800 & 35 & $21 \times 21$ & 85 & 3.5 \\
\hline
\end{tabular}

Fig. 3 shows the EUV spectra obtained in the 5 experiments for the indicated driver wavelengths. In the $400-\mathrm{nm}$ case the signal is much higher than in the other cases, so the peak is not shown. The "*" symbol shows the photon count number that has been considered for the wavelength study.

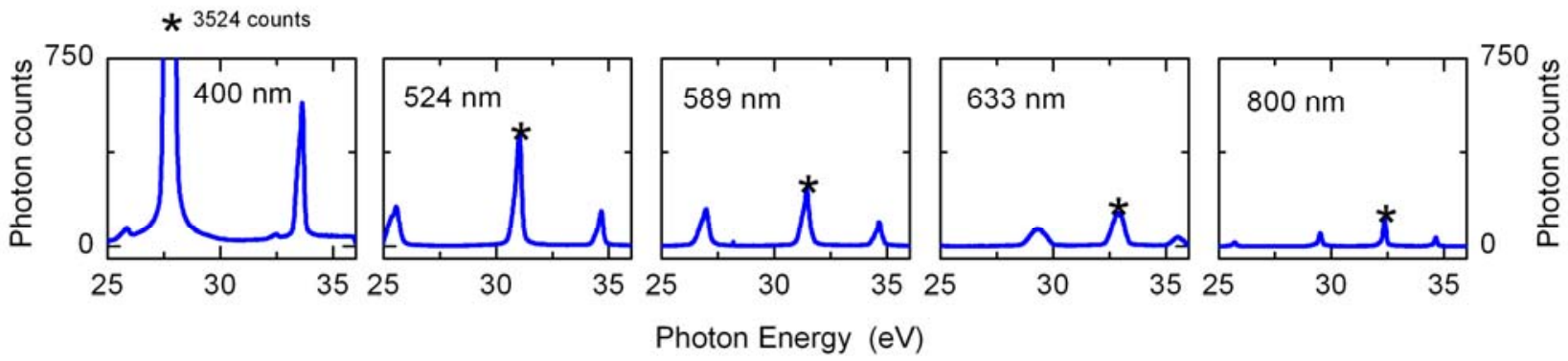

Fig. 3: HHG spectra for the 5 different wavelengths of the efficiency study (linear scale). 


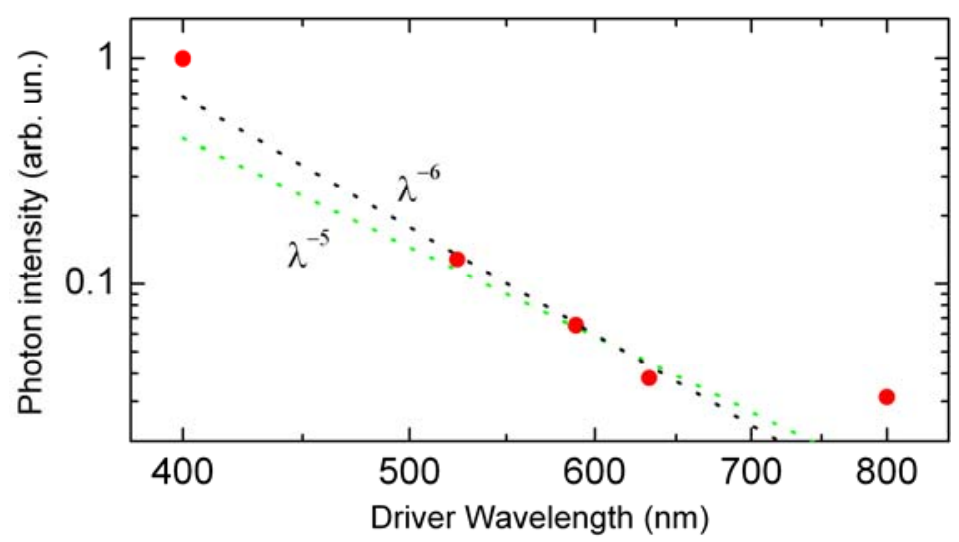

Fig. 4: Efficiency scaling in Argon vs. visible driver wavelength. (Red: experimental data). Black and green dots: theoretical predictions).

Fig. 4 shows the normalized number of photon counts observed in the spectrometer. As expected, the efficiency decreases as the wavelength increases. The result fits very well with the theoretical predictions, presented for example in [3], or [4], where an efficiency scaling of $\lambda^{-5} \div \lambda^{-6}$ is predicted. While the 3 experimental points observed with the OPA fit very well with the theoretical models, the two points at the fundamental frequency and at the second harmonic of the laser show a slightly higher efficiency. This might be due to the better beam quality in the laser beam than in the OPA, and also to their higher intensity, as indicated in Table 1.

\section{Conclusion}

In conclusion, we developed a tunable, high energy OPA in the visible range, starting from an amplified Ti:sapphire system with a 1-kHz repetition rate. We generate EUV pulses by driving HHG in Argon and, due to the broad tunability of our OPA, we are able to confirm efficiency scaling vs. driver wavelength in the visible wavelength range, thus experimentally verifying the theoretical predictions.

Other tunable sources like OPAs can be used to verify the driver wavelength dependence of HHG characteristics, like conversion efficiency and cutoff energy.

[1] P. Tzankov, J. Zheng, M. Mero, D. Polli, C. Manzoni, and G. Cerullo, “300 $\mu$ J noncollinear optical parametric amplifier in the visible at 1 kHz repetition rate," Opt. Lett. 24, 3629-3631 (2006).

[2] G. Cerullo and S. De Silvestri, “Ultrafast optical parametric amplifiers,” Rev. Sci. Instr. 74, 1-18 (2003).

[3] J. Tate, T. Auguste, H. G. Muller, P. Salières, P. Agostini, and L. F. DiMauro, "Scaling of Wave-Packet Dynamics in an Intense Midinfrared Field," Phys. Rev. Lett. 98, 013901 (2007).

[4] E. L. Falcão-Filho, V. M. Gkortsas, A. Gordon and F. X. Kärtner, "Analytic scaling analysis of high harmonic generation conversion efficiency,” Opt. Expr., 17, 11217-11229 (2009). 